\title{
LIGHTING IN CORRIDORS INFLUENCING THE SWITCHING ON OF ELECTRIC LIGHTING IN CLASSROOMS: NEED FOR EDUCATIONAL ACTIONS
}

\author{
AMERICO H. HARA ${ }^{1}$ \& FERNANDO O. R. PEREIRA ${ }^{2}$ \\ ${ }^{1}$ Santa Catarina State University, Centro de Educação Superior da Região Sul, Brazil \\ ${ }^{2}$ Federal University of Santa Catarina, Pos-Arq/UFSC, Brazil
}

\begin{abstract}
Studies suggest the lighting conditions in corridors can influence people's visual perception and, therefore, the use of electric lighting. The objective was to study the occurrence of switching on the electric lighting in classrooms due to the lighting conditions in the corridors and rooms. The methodology consisted of selecting corridors and classrooms that allow measurements of vertical eye illuminance measurements at $1.65 \mathrm{~m}$ height in the corridors and in the entrance door, and the registration of the switching on of the electric lighting in the classroom at the beginning of the occupation. For the first experiment $(\mathrm{N}=13)$, the vertical eye illuminance at the corridor was $80 \pm 3 \mathrm{~lx}$ and at the classroom door with $377 \pm 65 \mathrm{~lx}$, the switching on of the electric lighting in the classroom occurred in only $15 \%$. This indicates the switching on of the electric lighting was not significant when the corridor is darker than the classroom. For the second experiment $(\mathrm{N}=13)$, the vertical eye illuminance of the corridor was 4,185 $\pm 372 \mathrm{~lx}$ and, the classroom door was $253 \pm 24 \mathrm{~lx}$. Switching on of the electric lighting occurred in 54\%. There was significant activation when the corridor was brighter than the classroom. The third experiment $(\mathrm{N}=13)$ followed the same proposal as the second one, but with higher vertical eye illuminance values, where the corridor was $6,853 \pm 1,203 \mathrm{~lx}$ and at the classroom door, $880 \pm$ $75 \mathrm{~lx}$, the switching on of the lighting was $38 \%$. The results show that even if the classroom is sufficiently light, the switching on of the electric lighting occurred. Our conclusions describe that lighting in corridors greatly impact the perception of light in the classroom and interfere the switching on of the electric lighting, even with sufficient illuminance in classrooms, showing the need for education programs on energy efficiency and sustainability.

Keywords: user attitude, electric lighting, vertical eye illuminance, corridor, classroom.
\end{abstract}

\section{INTRODUCTION}

It is recognized that natural light has psycho-physiological impacts on the human being, as it is related to the regulation of the hormonal system, it offers the best color rendering, the perception of variability of intensity and tonality along the daytime and the luminous quality of the space through reflection in the surfaces [1]-[3].

Windows not just provide daylight into interior space but allow interior-exterior visual connection, and it is widely welcomed if natural elements are presented on the views [4].

Studies suggest that the incidence of natural light has the potential to save energy, since it can offer, in many cases, satisfactory lighting conditions in the internal space [5]. However, saving energy in buildings requires the user does not switch on the lights in the space when daylight is enough to work with visual comfort [6]. Therefore, it is critical to know how the user interacts with the artificial lighting system.

Previous works have identified the usual behavior was that all lights were turned on or would be off, and negligible the switching on some luminaires, at the arrival time. Besides, the lights were switched off only when the last person left the room [7]-[11].

Hunt [7] also found that in classrooms, the switch on and off activity of electric lighting occurred during all day, according to occupation. Hunt [8] correlated the minimum work plane illuminance from eqn (1) 


$$
y=-0,01754+\frac{(1,0364)}{(1+\exp (4,0835(\log \text { Ehmin }-1,8223)))},
$$

where $y$ is the probability of switch on the lights and Ehmin is the minimum work plane illuminance in the interior space.

According to the equation, the probability is null $(y=0)$ when the minimum illuminance in the horizontal plane is over $657 \mathrm{~lx}$ [8].

Studies suggest that only the illuminance in the horizontal plane is not sufficient to explain the switch on activity of the artificial lighting system by the user [12]-[14].

Particularly, Lynes et al. [13] support that the user's attitude of switch on electric light may be related to the pre-adaptation of the visual system to the lighting conditions in the corridor (transition space) and to the brightness (luminance) room surfaces.

Studies conducted by Shukuya and Morihana [15] have shown that sudden variations in light influence the visual perception of a space, that is, the sensation of bright and gloomy depends on the level of visual adaptation to the lighting conditions to which the observer was exposed previously. It is worth noting the application of vertical illuminance was used in research of visual perception considering certain position at a table in the room [16], [17], but studies of how visual adaptation in the corridor influence the activation or not of light in an occupation space are not developed.

This study aimed to investigate the activities of switching on electric lighting in classrooms, identifying whether partial or full system loaded, comparing with the Hunt's model probability [8] and, to relate with the lighting conditions in the corridors and at the entrance to the rooms.

\section{METHOLODOGY}

The experimental design supposes when the user walks down a corridor, his visual system will adapt to the lighting and, upon entering the room, he will experience a new situation, where he will assess the brightness of the room and will decide whether or not to switch on the lights.

The variables analyzed in this study were vertical eye illuminance, to characterize the amount of light in the plane of the observer's face, horizontal illuminance to calculate the probability of switching on the light based on eqn (1) and the switching on the part or the whole artificial lighting system.

Before starting the experiments, the researcher prepared the room by checking that the lights were off, and the curtains were all opened.

Initial measurements of vertical eye illuminance were performed in the corridor, at the classroom door and the horizontal illuminance, in the middle of the classroom, always before persons entered the room.

The participants walked the corridor (transition space) to the classroom entrance door, assessed the lighting condition and decided to whether the switch on the lights or not.

To carry out the experiments, three classrooms were selected with their corridors whose lighting conditions were different and which allowed light measurements. Each classroom with its corridor defined an experiment, called Exp-1, Exp-2 and Exp-3 (see Figs 1-3).

Measurements of vertical eye illuminance were conducted by using two supports to position the lux meters at 1.65 meters $(\mathrm{m})$ from the floor at points on Ev1 (corridors) and Ev2 (room doors). The horizontal illuminance (Eh) measurements were performed at $0.75 \mathrm{~m}$ from the floor, in the geometric center of each classroom, as showed in the floor plans (see Figs 1(a), 2(a) and 3(a)). 


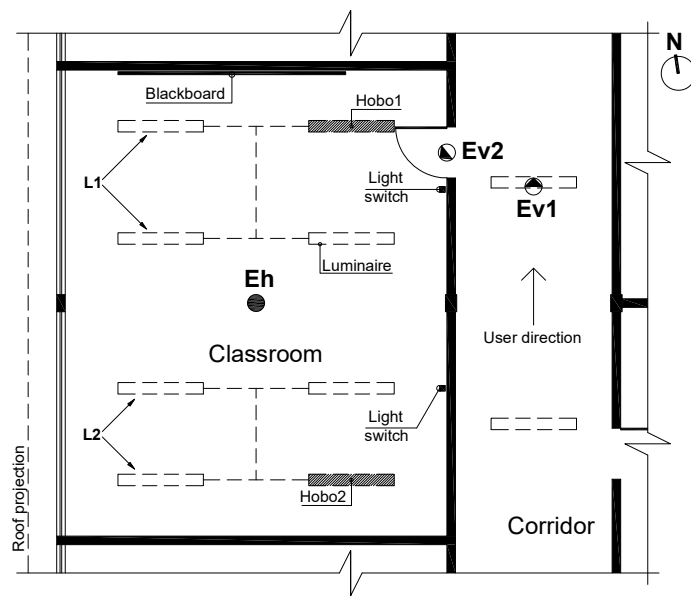

(a)

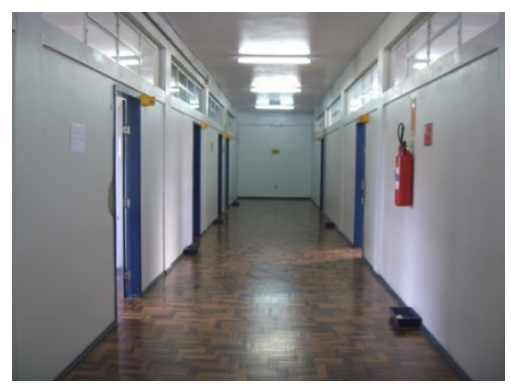

(b)

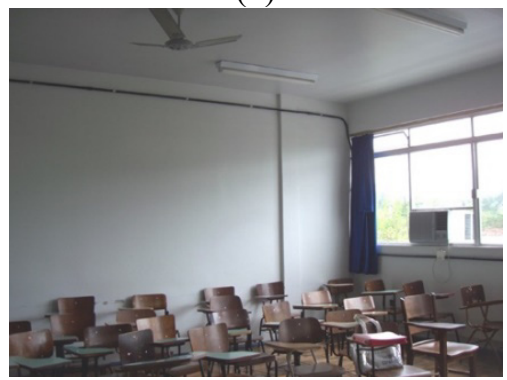

(c)

Figure 1: To Exp-1 in (a) The floor plan; (b) The corridor; and (c) The classroom.

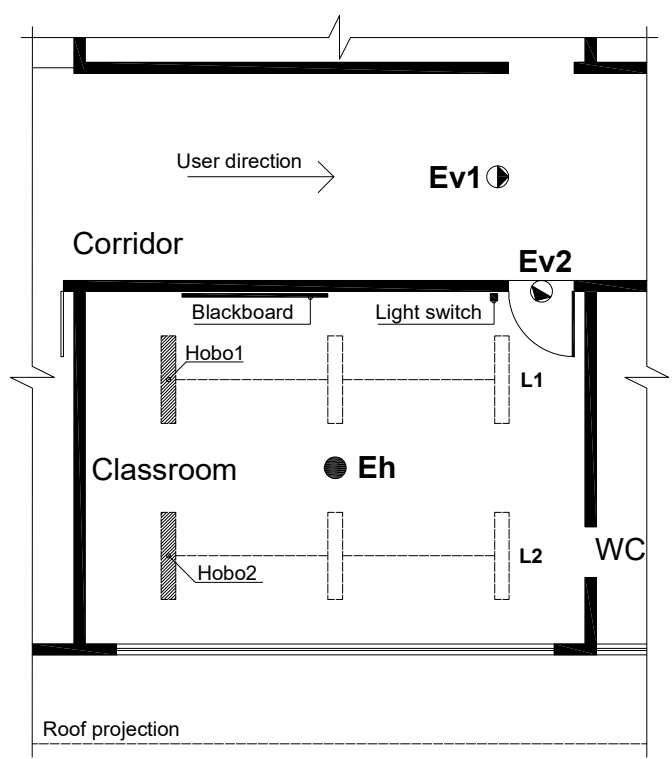

(a)

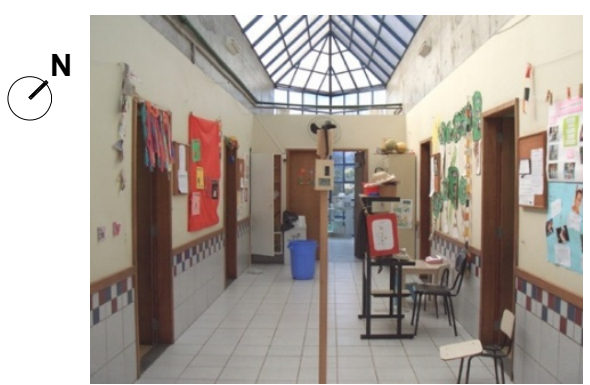

(b)

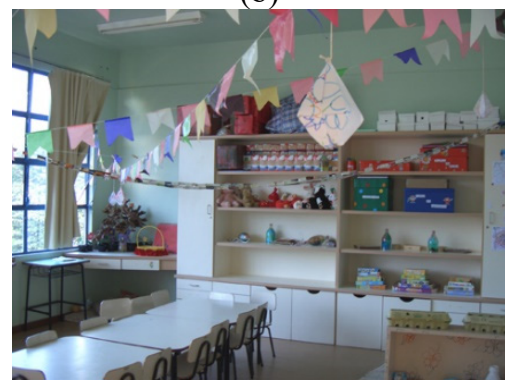

(c)

Figure 2: To Exp-2 in (a) The floor plan; (b) The corridor; and (c) The classroom. 


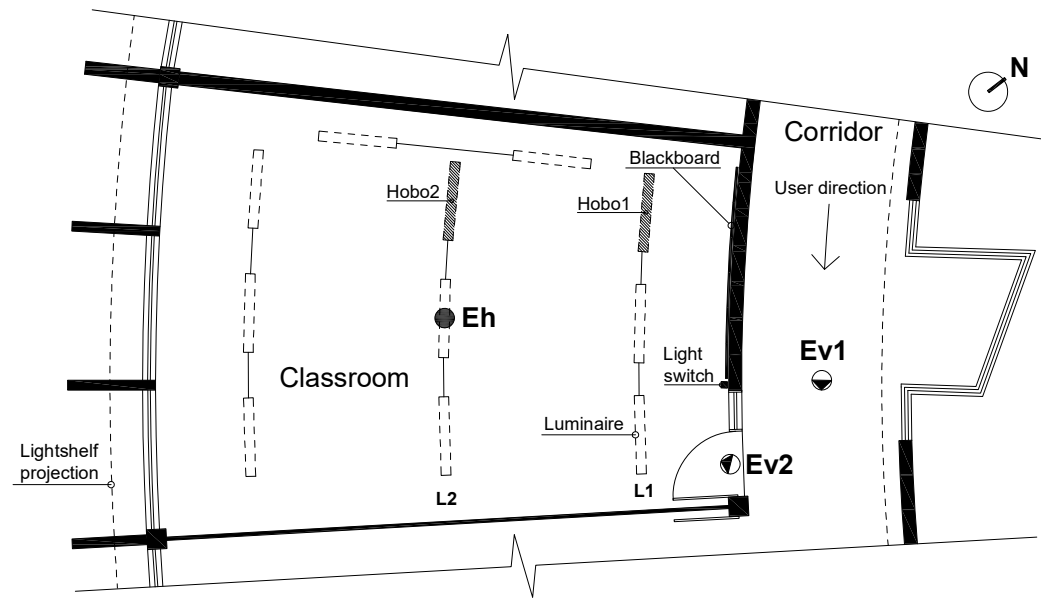

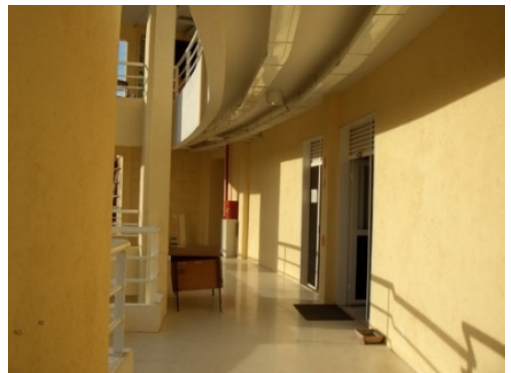

(b) (a)

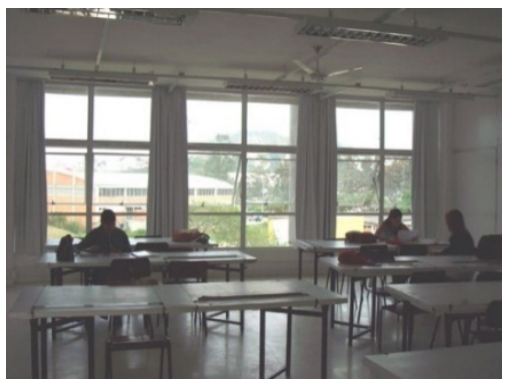

(c)

Figure 3: To Exp-3 in (a) The floor plan; (b) The corridor; and (c) The classroom.

The switch on the light was recorded by Hobos dataloggers light on/off installed in the luminaires, which made recordings every 0.5 seconds. The Hobos were placed in the space between the light and the luminaire so that they were hidden from users. The luminaires with hobos were denominated L1 and L2 (see Figs 1(a), 2(a) and 3(a)). The Hobos were installed before starting the measurements and removed after the end of each experiment.

In the Exp-1, the classroom access corridor is internal, illuminated basically with artificial light, whose illuminance on the floor is around $100 \mathrm{~lx}$ (Fig. 1(b)). The classroom had white walls and a dark wooden floor, west-facing windows and an area of 12.40 squared meter $\left(\mathrm{m}^{2}\right)$ and artificial lighting is controlled by two 2-button switches, one located near the entrance and other, on the same wall, toward the back of the room (Fig. 1(a) and 1(c)).

The corridor of the Exp-2 was over lit due to the skylight of polycarbonate transparent. On sunny days, the direct sunlight causes, in addition to thermal discomfort, visual discomfort, due to excess light (Fig. 2(a) and 2(b)). The classroom has green walls with a $12.00 \mathrm{~m}^{2}$ window facing the northeast, allowing natural light to enter in the morning (Fig. 2(c)). The artificial lighting is controlled by a 2-button switch located near the entrance to the room.

The corridor of the Exp-3 receives sunlight in the morning period and, because the floor is white, there are situations of glare caused by the reflection from the surface Fig. 3(a) and 3(b). The classroom has a white floor and walls, with south-west facing windows. Because 
the windows have a light shelf at the top of it, and because it occupies $76.1 \%$ of the wall, the room is well lit, and the distribution of daylight is quite uniform (Fig. 3(c)). The control of the artificial lighting system is made by a four-button switch located near the room entrance.

Table 1 summarizes the corridors and classrooms characteristics of this study.

Table 1: Characteristics of the corridors and rooms for the experiments Exp-1, Exp-2 and Exp-3.

\begin{tabular}{|l|c|c|c|c|c|c|c|c|c|}
\hline & \multicolumn{3}{|c|}{ Corridor } & \multicolumn{6}{c|}{ Classroom } \\
\cline { 2 - 10 } & $\begin{array}{c}\text { Lighting } \\
\text { type }\end{array}$ & $\begin{array}{c}\text { Width } \\
(\mathrm{m})\end{array}$ & $\begin{array}{c}\text { Height } \\
(\mathrm{m})\end{array}$ & $\begin{array}{c}\text { Room } \\
\text { area } \\
\left(\mathrm{m}^{2}\right)\end{array}$ & $\begin{array}{c}\text { Window } \\
\text { area } \\
\left(\mathrm{m}^{2}\right)\end{array}$ & $\begin{array}{c}\text { WWR } \\
(\%)\end{array}$ & $\begin{array}{c}\text { Window } \\
\text { orientation }\end{array}$ & $\begin{array}{c}\text { No. of } \\
\text { lumin. }\end{array}$ & $\begin{array}{c}\text { No. } \\
\text { of } \\
\text { lamps }\end{array}$ \\
\hline Exp-1 & Electric & 2.75 & 3.20 & 54.94 & 12.40 & 47.6 & W & 8 & 16 \\
\hline Exp-2 & Daylight & 2.85 & 3.50 & 33.22 & 12.00 & 54.7 & NE & 6 & 12 \\
\hline Exp-3 & Daylight & 2.35 & 3.00 & 55.41 & 15.51 & 76.1 & SW & 11 & 22 \\
\hline
\end{tabular}

Similar experiments were validated by the Human Research Ethics Committee of the Federal University of Santa Catarina, and the protocol number is 50831215.7.0000.0121CEPSH-UFSC, on 23 August 2016.

To prevent the use of curtains to block the sunlight inside the room, Exp-1 was conducted in the morning, starting at 07:30 a.m. until 12:00. Exp-2 and Exp-3 were performed in the afternoon, starting at 1:30 p.m. until 5:00 p.m. The experiments occurred on different days sequentially and were carried out in two consecutive weeks. None of the participants had any contact with each other. The research had 13 participants in each experiment in which: Exp$1 \mathrm{~N}=13$; Exp-2 $\mathrm{N}=13$, and Exp-3 $\mathrm{N}=13$.

The results are presented in graphs that indicate the switching on or off of the light in the classroom depending on the time after the individual has passed in a corridor, the evaluation of the lighting condition of the room by the users and during the activities in the classroom with the presence of the teacher.

Thus, room occupation intervals were defined to characterize the use of artificial lighting systems. The scheme in Fig. 4 presents two measurements: the entry period when students wait for the teacher in the classroom, and the class period after the teacher enters the room.

\begin{tabular}{|l|l|}
\hline Percentage of switch on the lights & \multicolumn{1}{|c|}{ Pime } \\
\hline $\begin{array}{l}\text { Students walk through the corridor and } \\
\text { enter the classroom. They can switch } \\
\text { on the lamps in the classroom. }\end{array}$ & $\begin{array}{l}\text { The professor and students in the } \\
\text { classroom can interact with artificial } \\
\text { lighting. }\end{array}$ \\
\hline
\end{tabular}

Figure 4: Flowchart 1 used for the experiments represented in the Figs 6 and 7.

The scheme showed in Fig. 5 has three measurements, because, the entry period was separate in two parts: first, the entry of the first user and his attitude over the artificial lighting system and, second, after the first user entry when other students enter the classroom previously the teacher. The third is still the class period. That distinction between the entrance 


\begin{tabular}{|l|c|c|}
\hline \multicolumn{1}{|c}{ Percentage of switch on the lights } & Percentage of switch on the lights & Percentage of switch on the lights \\
$\begin{array}{l}\text { The first student walks through the } \\
\text { corridor, enter the classroom and } \\
\begin{array}{l}\text { interact with the artificial lighting } \\
\text { system, soon after his entry. }\end{array}\end{array}$ & $\begin{array}{l}\text { Other colleagues walk through } \\
\text { the corridor, enter the } \\
\text { classroom and interact with } \\
\text { the artificial lighting system. }\end{array}$ & $\begin{array}{l}\text { The professor and students } \\
\text { in the classroom can interact } \\
\text { with artificial lighting. }\end{array}$ \\
\end{tabular}

Figure 5: Flowchart 2 used for the experiments represented in the Figs 8-12.

of the first user and the other is to observe how the lighting conditions of the empty room can influence the first user attitude without the interference of individuals present in the room.

\section{RESULTS AND DISCUSSIONS}

The results of the research are reported as follows: (i) The analysis of the results of the percentage of the interaction with the artificial lighting system according to the scheme described in Fig. 4; (ii) The comparison of the percentage of the switch on the light events with the percentage of probability calculated from eqn (1) according to Fig. 4; and (iii) The relationship between the switch on events carried out by the first user and the vertical eye illuminance in the corridor and the classroom as mentioned in Fig. 5.

\subsection{The use of artificial lighting system in the classroom}

In this work, it is necessary to explain that the lighting conditions were considered sufficient to carry out the activities, if the user does not switch on the lights.

The attitude of 13 participants in each experiment, Exp-1, Exp-2, and Exp-3, was analysed totalling a final sample of $\mathrm{N}=39$ events. Fig. 6 shows the results of the switch on the lights in the entry period and class period. In Exp-1, where the corridor is darker than the classroom, the percentage of people switch on the lights was the lowest compared to Exp-2 and Exp-3.

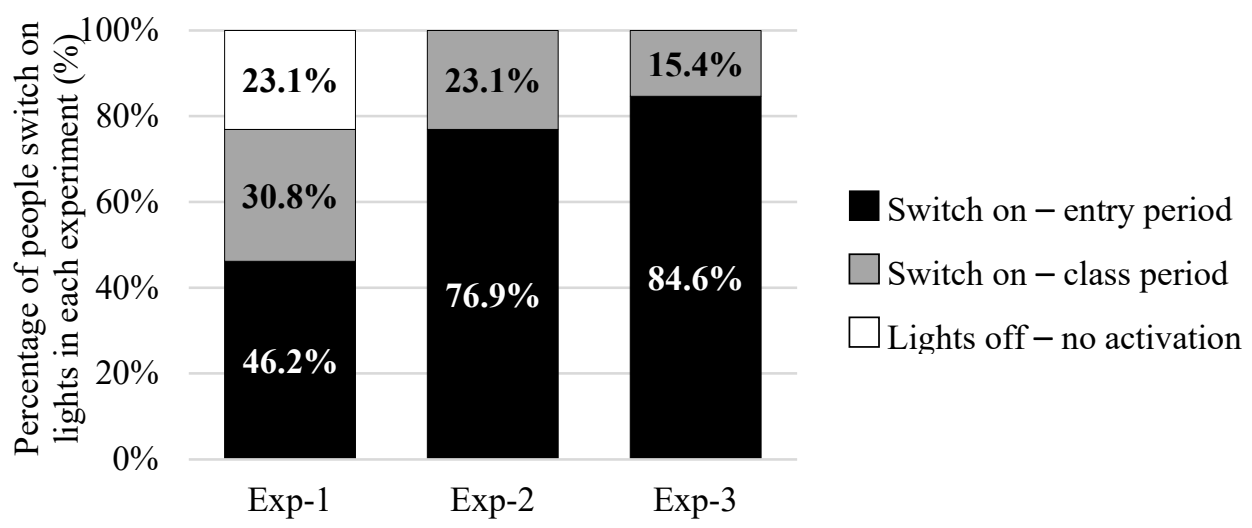

Figure 6: Percentage of people switch on the electric light: in black (entry period), the lights were switched on right after students entering the classroom; in grey (class period), the lights were switched on during the class period with the presence of the professor, and in white, when the lights remained off during all the occupation period (entry and class period). 
Besides, in Exp-1, during the entire period of occupation of the classroom, users do not switch on the lights in $23.1 \%$ of the events, whereas in Exp-2 and Exp-3, artificial lighting was activated in all events. Also, there was switching off the lights during the class period because of the activities, i.e. as to project lessons.

Table 2 details the type of switching on the lighting during the entry and the class periods, if partial (L1 or L2) or full (L1+L2). In Exp-1, part of lighting on (L1) was evident, both, in entry and class periods. In contrast, in Exp-2 and Exp-3, all the lighting system was on (L1 + L2), in both periods.

Table 2: Type of switching on the lighting system for the entry and class period in Exp-1, Exp-2 and Exp-3.

\begin{tabular}{|c|c|c|c|c|c|c|c|c|}
\hline LOCAL & \multicolumn{3}{|c|}{ Entry period - switch on lights } & \multicolumn{3}{c|}{ Class period - switch on lights } \\
\hline & L1 & L2 & L1+L2 & Total & L1 & L2 & L1+L2 & Total \\
\hline Exp-1 & $38.5 \%$ & $0.0 \%$ & $7.7 \%$ & $46.2 \%$ & $30.8 \%$ & $0.0 \%$ & $0.0 \%$ & $30.8 \%$ \\
\hline Exp-2 & $0.0 \%$ & $0.0 \%$ & $76.9 \%$ & $76.9 \%$ & $0.0 \%$ & $0.0 \%$ & $23.1 \%$ & $23.1 \%$ \\
\hline Exp-3 & $0.0 \%$ & $7.7 \%$ & $76.9 \%$ & $84.6 \%$ & $0.0 \%$ & $0.0 \%$ & $15.4 \%$ & $15.4 \%$ \\
\hline
\end{tabular}

The explanation for the occurrence of partial switching on lights in Exp-1 may be related to the location of the second switch away from the entrance door. The results obtained in Exp-2 and Exp-3 follow Hunt [7] and Love [9], observing that the users usually turn on all the lights at the beginning of the occupation.

In classrooms or space for collective use, the control of switching on the light can be operated in a neat way with the indication on the buttons the luminaires they activate. Another strategy is the placement of switches in two locations, one near the door to switch on the light near the entrance, and another, on the wall forward to the back of the room to turn on others one. Thus, it prevents the users to usually turn on all the lights, letting them adapt visually to the lighting conditions as they walk forward the back of the room.

Certainly, these suggestions have to be validated before their utilization, as they depend on the dimensions, the configuration of the room and the culture of people (i.e. habit). Also, educational actions are necessary for the artificial lighting system works as it was designed.

\subsection{The switching on artificial lighting in Exp-1, Exp-2, and Exp-3 compared}

to Hunt's [8] probability model

Values of the horizontal illuminance (Eh) was used to calculate the probabilities of switching on artificial lighting using the eqn (1) to be compared with the occurrences of the turned on the light during the entry period. The graphs in Fig. 7 shows the probability of switching on or off light with the hobos' registrations.

It is noteworthy that in Exp-1 and Exp-2 the probability of switching on the light is very similar, but the use of artificial lighting was different. In Exp-1, the light was on in $46.2 \%$ of events, and in Exp-2, 76.9\%. The difference is that the corridor in Exp-1 is darker than the room, and in Exp-2, it was the contrary. For the Exp-3, the probability is zero or very low because, the medium horizontal illuminances were $842 \pm 68$ lx, over $657 \mathrm{~lx}$, but the switch on events was the highest $(84.6 \%)$ of the three experiments.

The results obtained in the experiments suggest that only the horizontal illuminance is not sufficient to describe the manual switch on the light, as pointed out by some studies [12]-[14]. 

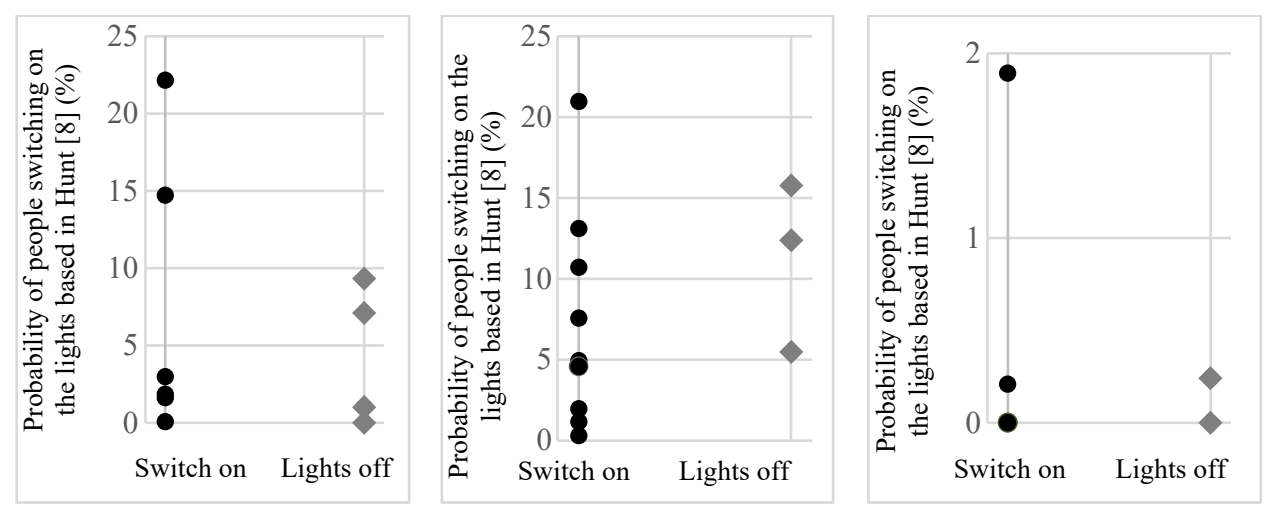

Figure 7: Probability of people switching on the lights based on eqn (1).

3.3 The switching on artificial lighting and the vertical eye illuminance in the corridor and the classroom.

A detailed analysis can be observed in the switching on the light by the first user who entered the classroom, as showed in the graph in Fig. 8.

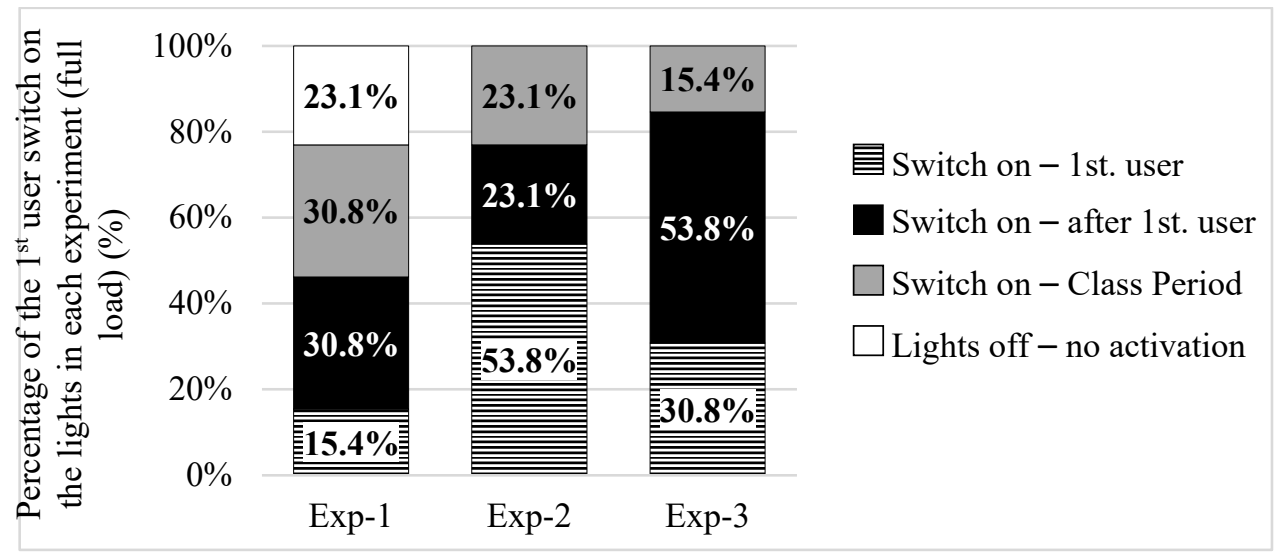

Figure 8: Percentage of people switch on the electric light. In horizontal lines hatch, the switching on the lights performed by the first user; in black (after first user entry), the lights were switched on after the first student entering the classroom; in grey (class period), the lights were switched on during the class period with the presence of the teacher, and white, when the lights that remained off.

A difference can be observed in the percentage of switching on the electric light between Exp-1, Exp-2, and Exp-3. To figure out these results, it is essential to verify the lighting conditions in the corridor and at the door soon after the first user entry. Vertical eye illuminance graphs can demonstrate these lighting conditions and help to explain the switching on the electric lighting from these dynamic changing (see Figs 9-12). 


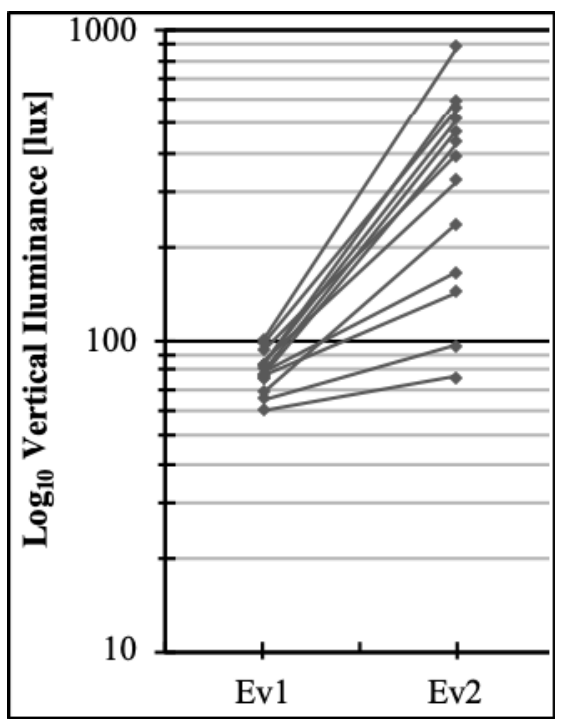

Figure 9: $\quad$ Exp-1 - vertical eye illuminance in Ev1 and Ev2.

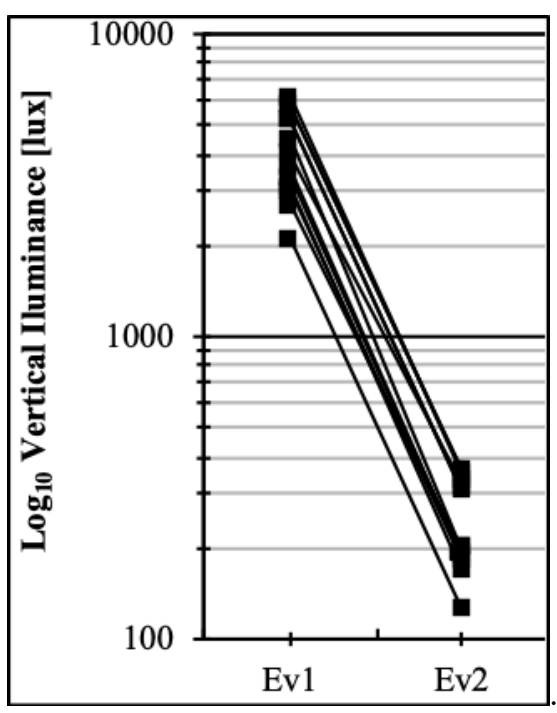

Figure 10: Exp-2 - vertical eye illuminance in Ev1 and Ev2.

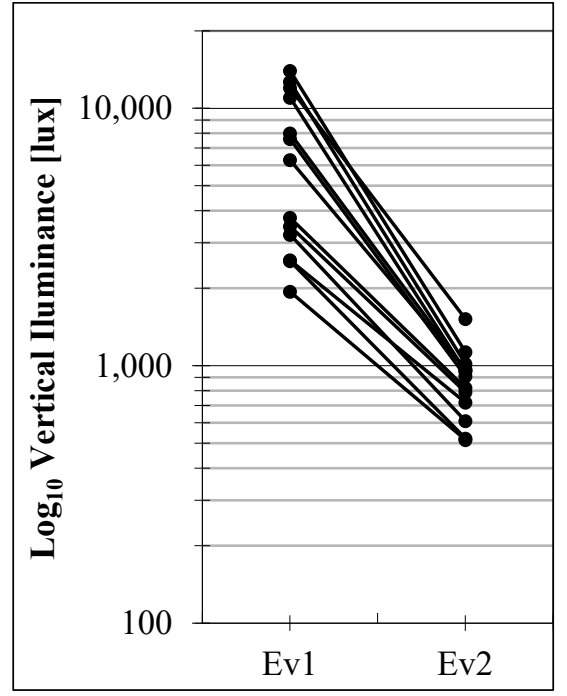

Figure 11: Exp-3 - vertical eye illuminance in Ev1 and Ev2.

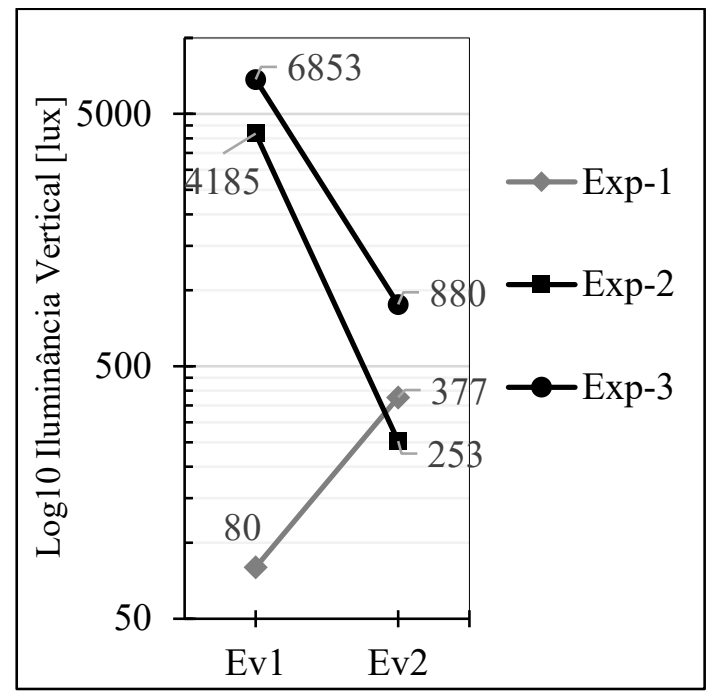

Figure 12: Medium vertical eye illuminance for Exp-1, Exp-2 and Exp-3.

In Exp-1, when the user passes through a corridor darker than the room, the switching on the light was less expressive (15.4\%). The average vertical eye illuminance in the corridor was $80 \pm 3 \mathrm{~lx}$, while in the classroom, it was $377 \pm 65 \mathrm{~lx}$ (see Fig. 9). This difference in 
vertical eye illuminance greatly influenced the switching on the light so the first user considered the room was sufficiently lit by daylight itself.

The graphs of Fig. 10 (Exp-2) and Fig. 11 (Exp-3) are similar, as the corridors are more lit than the classroom, as demonstrated in vertical eye illuminances, Ev1 and Ev2. In Exp-2, when the average vertical eye illuminance in the corridor was 4,185 $\pm 372 \mathrm{~lx}$ and at the door of the room, $253 \pm 24 \mathrm{~lx}$, the switching on events was $53.8 \%$, demonstrating that when the corridor is lighter than the classroom, there was more activation of electric lighting. The same was observed in Exp-3, where the vertical eye illuminance in the corridor was 6,853 $\pm 1,203$ $\mathrm{lx}$ and at the door, $880 \pm 75 \mathrm{~lx}$, the first user switched on the light in $30.8 \%$ of events, less than observed in Exp-2 (see Fig. 12).

However, the percentage of switching on light performed by the first user was significantly high in Exp-2 (53.8\%) compared to the Exp-3 (30.8\%). An explanation can be the vertical eye illuminance on the room door, while in Exp-2, was $253 \pm 241 x$, in the Exp-3, was $880 \pm 751 x$.

In Exp-3, although the user has experienced high amounts of lighting in the corridor, when he arrives at the door, he realizes that the space is bright. In contrast, in Exp-2, whose percentage of switching on the light was significant, lighting is not enough for a satisfactory visualization of the environment. It seems that the vertical eye illuminance at these two points can describe the relationship between the activation of artificial lighting and the visual perception of the room.

\section{CONCLUSIONS}

The study analyzed the user's attitude over the artificial lighting system in three classrooms considering the visual adaptation of users in the corridor, and we observed that the switching on all the lights occurred, mainly during the entry period.

However, the partial activation observed in Exp-1 is due to the switches layout, one at the entrance and other forward the back of the room, rather than to the users' consciousness of energy use in lighting. This indicates that, in some cases, the switches layout can be an alternative for conscious use of artificial lighting.

Although the horizontal illuminance is a parameter to calculate the probability of switching on the lights based on Hunt's equation (eqn (1)), we observed discrepancies with the experiments results. This discrepancy indicates that only the horizontal illuminance is not sufficient to describe the switching on the light, as reported in [12]-[14].

The study revealed that even with sufficient illuminance in classrooms as recommended by the standards, people preferred to switch on the light, showing the need to promote educational actions on energy efficiency and sustainability, in addition to more studies on visual perception and visual discomfort.

Considering just the first user, it was possible to describe how the lighting condition in corridors influenced in the switching on the light in the room. There are indications that vertical eye illuminance in these two points, can help to describe the relationship between the switching on the electric lighting and the visual perception of the room. Further studies are needed to correlate vertical eye illuminance, the manual activation of electric lighting and visual perception from the door point of view.

\section{ACKNOWLEDGEMENTS}

Thanks are due to the management and staff of the corridors and classrooms, and the head of the professors, professors and students at the Federal University of Santa Catarina buildings concerned to co-operating with the studies. Thanks to the Brazilian National Council for Scientific and Technological Development $(\mathrm{CNPq})$, and the Santa Catarina State University 
for the support in developing this work. Thanks to F. O. R. Pereira for the assistance and supervisory.

\section{REFERENCES}

[1] Baker, N., Cultural responses to primitive needs. WIT Transactions on the Built Environment, vol. 86, WIT Press: Southampton and Boston, pp. 3-13, 2006.

[2] Patania, F., Gagliano, A., Nocera, F., Galesi, A. \& Caserta, J., The dynamic lighting into the working environment. International Journal of Design and Nature and Ecodynamics, 7(4), pp. 394-408, 2012.

[3] Pennisi, S., Scaccianoce, G. \& Vaccaro, V. Indoor comfort in school buildings: A case study, Palermo, Italy. WIT Transactions on Ecology and the Environment, vol. 191, WIT Press: Southampton and Boston, pp. 1685-1696, 2014.

[4] Shin, J.Y., Yun, G.Y. \& Kim, J.T., View types and luminance effects on discomfort glare assessment from windows. Energy and Buildings, 46, pp. 139-145, 2012.

[5] Bourgeois, D., Reinhart, C. \& Macdonald, I., Adding advanced behavioural models in whole building energy simulation: A study on the total energy impact of manual and automated lighting control. Energy and Buildings, 38(7), pp. 814-823, 2006.

[6] Leslie, R.P., Capturing the daylight dividend in buildings: Why and how? Building and Environment, 1(38), pp. 381-385, 2003.

[7] Hunt, D.R.G., The use of artificial lighting in relation to daylight levels and occupancy. Building and Environment, 14, pp. 21-33, 1979.

[8] Hunt, D.R.G., Predicting artificial lighting use: A method based upon observed patterns of behaviour. Lighting Research and Technology, 12(1), pp. 7-14, 1980.

[9] Love, J.A., Manual switching patterns in private offices. Lighting Research and Technology, 30(1), pp. 45-50, 1998.

[10] Reinhart, C.F. \& Walkenhorst, O., Validation of dynamic RADIANCE-based daylight simulations for a test office with external blinds. Energy and Buildings, 33(7), pp. 683697, 2001.

[11] Silva, P.C., Leal, V. \& Andersen, M., Occupants interaction with electric lighting and shading systems in real single-occupied offices: Results from a monitoring campaign. Building and Environment, 64, pp. 152-168, 2013.

[12] Assaf, L.O. \& De Wilde, M.I., Un procedimiento para mensurar la contribución energética efectiva del alumbrado natural en edifícios. Proceedings of LUXAMERICA 2000, São Paulo, 2000.

[13] Lynes, J.A., Littlefair, P.J. \& Slater, A.I., Pre-adaptation and manual switching. Proceedings of Right Light 4, 2, pp. 219-224, 1997.

[14] Yun, G.Y., Kim, H. \& Kim, J.T., Effects of occupancy and lighting use patterns on lighting energy consumption. Energy and Buildings, 46, pp. 152-158, 2012.

[15] Shukuya, M. \& Morihana, T., Variations of daylight and electric light and the associated brightness sensation: For the possible use as a passive strategy. Proceedings of PLEA, 17, pp. 644-645, 2000.

[16] Suk, J.Y., Luminance and vertical eye illuminance thresholds for occupants' visual comfort in daylit office environments. Building and Environment, 148, pp. 107-115, 2019.

[17] Van Den Wymelenberg, K. \& Inanici, M., A critical investigation of common lighting design metrics for predicting human visual comfort in offices with daylight. LEUKOS - Journal of Illuminating Engineering Society of North America, 10(3), pp. 145-164, 2014. 